\title{
Carlos Emilio García Duque*
}

\section{¿Quién le teme a la filosofía para niños? Who's afraid of Philosophy for children?}

Porque el niño no es capaz de distinguir donde se da o no la alegoría, y todo lo que recibe en su alma a tal edad, difícilmente se borra o se cambia. Por lo cual, seguramente convenga antes de nada que las primeras fábulas que oiga el niño sean también las más adecuadas para conducirle a la virtud.

Platón, República 2-XVII, 379b

\begin{abstract}
Resumen. El objetivo central de este trabajo es caracterizar el programa de Filosofía para niños a partir del análisis de la consistencia de sus planteamientos y la presentación de ciertos aspectos del programa que ciertamente pueden ser desarrollados con mayor produndidad. La idea es extender una invitación a iniciar un debate crítico sobre une tema que cada vez cobra más fuerza entre profesores y estudiantes universitarios de filosofía.
\end{abstract}

Palabras clave: filosofía para niños, Mathew Lipman, enseñanza de la filosofía.

Abstract. The central aim of the present work is to characterize the Philosophy for Children Program from the analysis if its consistency and the presentation of certain aspects of the program that certainly could be developed more in depth. The idea is to extend an invitation to initiate a critical debate about a topic that each day gains more and more importance among college teachers and students.

Key words: philosphy for children, Mathew Lipman, philosophy education.

\section{Introducción}

A finales de la década de los sesenta, Matthew Lipman y sus colaboradores crearon el programa de Filosofía para niños (en adelante FpN) como una propuesta innovadora que buscaba, entre muchos otros fines, trasladar una parte sustancial de la actividad filosófica de los claustros universitarios a las aulas de clase de las escuelas, generando de paso una especie de revolución educativa, encaminada a corregir muchos de los yerros y fracasos del pasado $^{1}$. En las últimas décadas, este proyecto ha tenido muy buena acogida en países como España, México, Brasil y más recientemente en Colombia, donde acaba de finalizar el tercer Congreso Latinoamericano sobre el tema. A juzgar por lo que dice

* Profesor de la Universidad de Caldas.

cegarcia@um.umanizales.edu.co

Cf. Lipman, Matthew, Sharp Ann M. y Oscanyan, Frederick S. La Filosofia en el Aula, Madrid, Ediciones de la Torre, 1992. 
la literatura al respecto y por lo que se escucha en los departamentos de filosofía de nuestras universidades, el entusiasmo con que algunos educadores y ciertas instituciones de enseñanza (sobre todo en el nivel básico y medio) han recibido la propuesta de Lipman no guarda proporción con el escepticismo o el poco conocimiento de ella, que parecen ser la constante entre quienes se dedican profesionalmente al cultivo de la filosofía. El hecho de que profesores universitarios experimentados que han dedicado toda su vida a la práctica y la enseñanza de la filosofía expresen serias dudas sobre la posibilidad misma de que un proyecto tal pueda siquiera operar, constituye un indicador de cuan urgente resulta examinar el asunto con cuidado. En este trabajo me propongo caracterizar muy brevemente el programa de FpN, explorar la consistencia de sus planteamientos, hacer algunas precisiones sobre ciertos aspectos de la propuesta que considero insuficientemente desarrollados, e invitarlos a iniciar un debate crítico más informado y mejor estructurado sobre un tema que me parece requiere de atención inmediata de parte de profesores y estudiantes universitarios de filosofía.

Pese a que mi ensayo carece de una tesis central, he de presentar tres conjeturas sobre el estado de la cuestión. La primera de ellas es que aunque el programa de $\mathrm{FpN}$ resulta interesante, atractivo e innovador en un campo que, como el de la educación, requiere con urgencia de ideas refrescantes y propuestas audaces, la manera como se han defendido sus tesis centrales es francamente insatisfactoria. En segundo lugar, he de sugerir que, luego del análisis de rigor, la expresión "filosofía para niños" resulta demasiado ambigua como para ser de utilidad en una discusión seria. Es lo que se conoce como una falsa etiqueta (misnomer) que puede confundir al lector tentándolo a extraer las conclusiones erradas. Por último, he de advertir que el programa de FpN es particularmente vulnerable a uno de los mayores riesgos que enfrenta la formación en áreas como las sociales y humanísticas, a saber, la proliferación indiscriminada de verborreas y la mediocridad académica, disfrazadas con el ropaje deslumbrante de objetivos educativos con los que todos estamos de acuerdo; como por ejemplo, los del desarrollo de habilidades superiores de pensamiento, destrezas analíticas, valores democráticos y otras características que consideramos deseables en una formación de buena calidad, compatible con principios como los de libertad y democracia.

\section{Filosofía para niños}

Según Pineda, el principal divulgador de este programa en nuestro país, filosofía para niños es un "un proyecto pedagógico de alcance mundial cuya pretensión básica es la de articular la educación del futuro, en torno al desarrollo del pensamiento superior en sus diversas modalidades (pensamiento analítico, intuitivo, crítico, reflexivo, creativo, etc.), y que se apoya en la introducción de la reflexión filosófica en todos los niveles y temáticas del currículo" ${ }^{2}$. Esta ambiciosa propuesta sostiene que "los niños necesitan tanto de la filosofía como los adultos" y que es posible introducir activa y exitosamente la filosofía desde la escuela primaria, permeando prácticamente todas las asignaturas con que ha de enfrentarse el estudiante durante su experiencia escolar. Para sacar adelante sus objetivos, el programa plantea un mecanismo de trabajo colectivo muy interesante que se denomina "comunidad de indagación", un espacio de trabajo en el que los niños aprenden las reglas del juego de la filosofía, al tiempo que desarrollan habilidades de razonamiento y metacognición, y someten a la crítica sus argumentos e intuiciones filosóficas. Con respecto a la dicotomía entre quienes destacan el aspecto instrumental de la filosofía (mediante ella es posible animar a los niños a que sean más reflexivos y críticos en su contacto con otras asignaturas del currículo) y quienes defienden la preservación de la integridad de la filosofía como disciplina, para

2 Pineda, Diego Antonio. "Filosofía para niños: un Proyecto de Educación Filosófica". En: Cultura, Separata No. 3, 2003, pp. 1-20 
explotar sus virtudes únicas (estimular la curiosidad y contribuir al desarrollo del carácter reflexivo), los partidarios de FpN sostienen que esta es una falsa dicotomía que puede ser superada teniendo en cuenta que las experiencias de quienes han empleado este programa indican cómo los niños a quienes se ha "enseñado a ser sistemáticamente curiosos y naturalmente reflexivos, tienden a extender esa conducta al resto de sus aprendizajes."

El programa de FpN tiene cuatro objetivos y dos meta-objetivos (al decir de Pineda). Los objetivos son: 1. El desarrollo de la capacidad de razonamiento mediante el uso de diversas lógicas (formal, informal y lógica del actuar racional), al tiempo que se atiende a las necesidades de la vida cotidiana, del trabajo intelectual, de la acción moral, de la creatividad y de las habilidades propias para la vida en sociedad; 2. El desarrollo de la comprensión ética al margen de adoctrinamientos dogmáticos y concesiones al relativismo moral. En la consecución de este objetivo, la $\mathrm{FpN}$ proporciona las herramientas para identificar situaciones morales, evaluarlas de manera adecuada y examinar dichas valoraciones con criterios generales; 3 . El desarrollo de la capacidad para descubrir significado en la experiencia, para lo que es necesario aprender a reconocer semejanzas y diferencias, así como a establecer relaciones y a participar en juegos de lenguaje que requieren el empleo de estrategias mentales; 4. El desarrollo de la creatividad "ligada a la capacidad de desarrollar modos alternativos de pensamiento", bajo el común denominador del trabajo lógico, el que, según los creadores del programa, proporciona la clave esencial para conseguir tan importante logro.

Los dos meta-objetivos del programa son: 1 . El crecimiento personal e interpersonal, como un resultado hipotético de una mejor auto-comprensión y una conciencia adecuada de las actitudes, emociones e intereses de cada individuo; y 2. La formación de valores democráticos para la

3 Lipman, et. al. op. cit., p. 116. convivencia ciudadana mediante la educación de individuos capaces de pensar correctamente por sí mismos, y preparados adecuadamente para examinar de manera racional sus creencias por medio de la confrontación con sus compañeros y maestros en la institución escolar ${ }^{4}$.

\section{Despejando las objeciones convencionales}

Como es de esperar, Lipman y sus discípulos comienzan por responder a las objeciones que se pueden formular a un proyecto como el de la FpN desde tres disciplinas independientes pero que históricamente estuvieron estrechamente relacionadas, a saber: la filosofía, la psicología y la pedagogía. Presentaré estas objeciones en el mismo orden en que las enuncié. La historia de la filosofía muestra que las ideas filosóficas más perdurables e influyentes fueron creadas por personas relativamente maduras que habían dedicado largos años de su vida a reflexionar profundamente sobre las cuestiones en torno a las que giraban sus teorías. Más aún, consideraciones críticas y hermenéuticas bastante comunes nos persuaden no sólo de que es posible descubrir la evolución del pensamiento filosófico de muchos autores (cuyas ideas se tornan más claras y mejor estructuradas después de años de trabajo), sino también identificar los puntos de ruptura y revolución mediante los cuales un pensador abandona las tesis de sus maestros para comenzar a pensar por cuenta propia ${ }^{5}$. Si la práctica de la filosofía tiene algo que ver con el pensamiento original, no parece

\footnotetext{
4 He resumido estos objetivos del artículo de Pineda, op. cit., pp. 9-10.

5 Dejo de lado deliberadamente la discusión acerca de si se puede hacer filosofía sin estudiar la historia de la filosofía o sin estar debidamente familiarizado con los problemas y las teorías propias del pensamiento filosófico. Esta posición es vulnerable a objeciones que señalan (correctamente) que la familiaridad con la filosofía, con sus temas y sus problemas, no es una condición suficiente ( $\mathrm{y}$ tal vez tampoco sea necesaria) para hacer filosofía. Por otra parte, queda pendiente una caracterización precisa de lo que significa "hacer filosofía" desde el marco de referencia de la FpN, punto en que los creadores del programa no han sido suficientemente claros.
} 
posible que los niños se involucren en esta clase de actividades desde su más tierna edad, con posibilidades realistas de salir airosos ${ }^{6}$. Además, la misma objeción se sostiene si se acepta que para crear filosofía se requiere el dominio de un repertorio lingüístico y conceptual mínimo, que por definición no podría estar al alcance de estos alumnos.

La psicología evolutiva, tal como fue desarrollada por Piaget y sus seguidores, sugiere que, en su desarrollo psicológico, los seres humanos atraviesan por una serie de etapas que tienen profundas repercusiones en la evolución de su pensamiento y en la potenciación de su capacidad para lograr aprendizajes significativos sobre temas de diversos grados de complejidad y dificultad. Si esta teoría es correcta, aunque educadores y discípulos pongan lo mejor de sí en la tarea, la edad de los segundos resulta ser una variable importante que puede facilitar o entorpecer de manera decisiva el desarrollo de ciertas habilidades y la consecución de determinadas metas. Por esta razón, alumnos sumamente jóvenes podrían fracasar al momento de embarcarse en actividades complejas que requerirían de la posesión de ciertas destrezas que ellos, debido al estado de su desarrollo psicológico, no poseen por no haberlas desarrollado aún. Por lo tanto, muchos niños, por ejemplo los que no han llegado a la etapa del pensamiento lógico y conceptual, simplemente no podrían hacer filosofía. Por último, las teorías instruccionales y las prácticas pedagógicas establecidas en nuestros sistemas de enseñanza, recomiendan la presentación de contenidos y el desarrollo de habilidades complejas en un orden que revela la aceptación de las tesis de la psicología evolutiva, por un lado, y del sentido común, por el otro. Unos y otras deben ordenarse según principios que van de lo simple a lo complejo y que tienen en cuenta las relaciones e interrelaciones que pueden establecerse entre los elementos de un sistema. Por ejemplo, los conceptos se presentan en orden de complejidad creciente (con la debida

\footnotetext{
6 El programa de $\mathrm{FpN}$ sostiene que debe comenzarse a hacer filosofía desde el preescolar, con niños de tres años de edad.
}

consideración de sus relaciones de dependencia) y las habilidades de pensamiento se desarrollan de manera parecida, teniendo siempre en mente la imagen de modelos altamente estructurados.

Como se supone que la filosofía corresponde precisamente a una actividad intelectual altamente estructurada y compleja, que requiere de conceptos muy abstractos y, por tanto, difíciles de adquirir, la organización de los saberes en el sistema escolar ha privilegiado la idea de que es conveniente diferir la introducción de la filosofía hasta los últimos años de la educación secundaria o los primeros de la universitaria. En resumen, la naturaleza compleja y difícil de la filosofía y el hecho de que por su carácter abstracto tiende a ser percibida como un tema de estudio aburrido -o cuando menos inapropiado para pupilos muy jóvenes-sugieren que la filosofía no se puede presentar con provecho a los niños. Debido a esto, la pedagogía corriente no vería con muy buenos ojos a un programa como el que encarna la FpN. Veamos ahora la forma como responden a estas objeciones Lipman y sus seguidores.

La respuesta a las objeciones provenientes de la filosofía consiste en aclarar que el objetivo del programa de $\mathrm{FpN}$ no es enseñar filosofía $a$ los niños sino hacer filosofía con los niños ${ }^{7}$. Por esta razón, los defensores del programa no tienen que preocuparse por la naturaleza compleja y el carácter abstracto de la filosofía. El estereotipo de la filosofía como una actividad "aburrida" a la que sólo se pueden dedicar los adultos tampoco resulta ser una buena objeción. Como bien se sabe, incluso los iniciadores de la filosofía en la antigüedad clásica reconocieron en la curiosidad y la capacidad de asombro su punto de partida; y si se trata de hallar individuos bien dispuestos hacia el asombro y la

\footnotetext{
7 Marcela Blanco insiste en algo parecido, aunque ella es partidaria de modificar el para del programa original y transformarlo en con. Infortunadamente su ensayo es todavía muy elemental como para poder apreciar las implicaciones de esta propuesta. Cf. Blanco, Marcela. "Filosofía con niños". En: Filosofia, pedagogía y enseñanza de la filosofia. Germán Vargas y Luz Gloria Cárdenas, Bogotá: Universidad Pedagógica Nacional, 2004, pp. 365-369.
} 
curiosidad, sin lugar a dudas los niños son los mejores candidatos. Por otra parte, es falso que algunos filósofos griegos hayan prohibido explícitamente la enseñanza de la filosofía a los niños. Lipman se esfuerza por examinar e interpretar de manera favorable un pasaje de la República de Platón donde puede encontrarse una opinión en este sentido. De acuerdo con Lipman, Platón no estaba en contra de la enseñanza de la filosofía a los niños, sino más bien en contra de la creencia de que enseñar filosofía era equivalente a enseñar retórica ${ }^{8}$. Sin embargo, la respuesta más contundente a esta clase de objeciones parece provenir de la experiencia: los niños son capaces de considerar asuntos filosóficos (e.g. la belleza, la bondad, la justicia, etc.) y de resolver problemas relacionados con estos temas. Además, en la medida en que pueden formular y responder preguntas, y sobre todo, en tanto están dispuestos a examinar críticamente las implicaciones de sus supuestos, y a evitar falacias o a alejarse de los razonamientos incorrectos, demuestran que pueden hacer filosofía.

Por lo que respecta a las objeciones de la psicología, los creadores de FpN parecen sugerir que las teorías sobre el desarrollo evolutivo del pensamiento son falsas. Si bien no ofrecen una discusión estructurada del problema, acuden a argumentos empíricos (que dicho sea de paso, de ser correctos constituirían una refutación de las teorías involucradas) que presumiblemente indican que no hay motivos para diferir hasta la adolescencia o los últimos años de la educación secundaria, la enseñanza o, cuando menos, la introducción de actividades destinadas a promover el desarrollo de habilidades superiores de pensamiento. Algo parecido ocurre con las objeciones provenientes de la pedagogía. En opinión de los creadores de la $\mathrm{FpN}$, las prácticas convencionales de organización y secuenciación de contenidos en realidad reproducen

8 Cito un fragmento del texto discutido por Lipman: “(...) cuando [los niños] han recibido las primeras lecciones de dialéctica se sirven de ella como un pasatiempo, y disfrutan provocando controversias sin cesar". Platón. ideas inadecuadas sobre la naturaleza del saber y promueven errores como la presentación aislada y compartimentalizada del conocimiento.

\section{Lugares comunes y promesas desmesuradas}

Creo que uno de los puntos más débiles del programa de FpN radica en el carácter trivial de buena parte de lo que constituyen sus motivaciones pedagógicas y los argumentos para la defensa de sus logros. En efecto, durante los últimos cincuenta años hemos estado discutiendo, hasta el cansancio, sobre la importancia de mejorar la calidad de la educación, de formar personas analíticas, críticas, capaces de pensar de manera independiente y creativa, y dispuestas a conservar y reproducir los valores que consideramos importantes para el fortalecimiento de una sociedad libre, democrática, abierta y tolerante. Cuando los partidarios del programa de FpN nos dicen que es importante formar el espíritu crítico de los niños, o intentan persuadirnos de cuan beneficioso resulta el cultivo de habilidades que aseguren el pensamiento correcto, ¿no nos están repitiendo acaso un sermón de cuya moraleja hace mucho tiempo todos estamos convencidos? Por supuesto que estos objetivos son tan importantes como deseables y nadie, en su sano juicio, se opondría a un programa que mejore la situación en estos aspectos, pero una cosa es plantear ideas abstractas sobre las que hay acuerdos casi universales y otra muy distinta, mostrar que la FpN constituye el medio más adecuado para lograr estos propósitos, o establecer que, en efecto, los consigue.

Consideren, por ejemplo, el punto central del programa, a saber la caracterización de la filosofía, el rol que se le atribuye en el proceso educativo y lo que significa la expresión "hacer filosofía con los niños". Lipman y sus seguidores aseguran que cuando los niños, que participan en comunidades de indagación, se involucran en ciertas actividades están haciendo filosofía. Así, los niños deben comprometerse: 
(...) con el planteamiento de preguntas relevantes, con la identificación de los supuestos subyacentes a sus afirmaciones, con la previsión de las consecuencias posibles de sus enunciados y sus actos mentales, con el ofrecimiento y evaluación de razones, con el análisis del lenguaje que utilizan, con la revisión de sus razonamientos, con el planteamiento de hipótesis teóricas y con su contrastación mediante ejemplos y contraejemplos, etc ${ }^{9}$.

No podría encontrar una lista de desiderata con la que pudiera estar más de acuerdo. Pero, ¿por qué llamar a estos compromisos filosofía? Parece haber aquí una confusión entre lo filosófico y la filosofía. Todas las actividades incluidas en el texto citado hacen parte del método de la filosofía; pero, como es obvio, la naturaleza de un método no se transfiere a los productos obtenidos mediante su aplicación. Por otra parte, sólo a través de una definición tremendamente deflacionaria de la filosofía, podríamos hacer pasar por filosofía lo que resulta de la aplicación de sus métodos a ciertas temáticas que se le parecen sólo de manera accidental. De otro modo, habría que revisar el uso del vocablo y aceptar que siempre que una persona se involucra en actividades intelectuales con ciertos estándares mínimos de racionalidad está haciendo filosofía. Sin embargo, nos guste o no, el calificativo de "filosófico" es uno que no suele dispensarse con tanta liberalidad como parece demandar el proyecto de FpN.

Una dificultad similar surge al momento de caracterizar la categoría de "niño". Hace ya algún tiempo que los profesores de filosofía de colegios y universidades trabajan con niños entre los 14 y los 16 años. Desde este punto de vista, la FpN no sería una práctica nueva en absoluto. Para complicar las cosas, Diego Pineda escribe en su exposición del tema que la FpN se puede trabajar desde el preescolar hasta el postgrado porque el termino "niño" no debe entenderse en sentido cronológico sino

9 Pineda, Diego A. op. cit., p. 3. que designa a

...todo aquel que dispone de algo que es esencial al niño y que ha sido considerado desde siempre como el punto de partida del filosofar original: la capacidad de asombro ${ }^{10}$.

Por eso concluye que la FpN debe estar dirigida sobre todo a los adultos y buscar en especial que los maestros puedan recuperar el placer connatural al ejercicio del pensamiento. Pero si esta afirmación ha de ser la interpretación correcta del asunto, queda poco de lo que constituye el planteamiento más revolucionario del programa. Considero que el profesor Pineda se dejó llevar aquí de su desmedido entusiasmo y que extendió de manera abusiva la metáfora de la curiosidad y el asombro (elementos sin los cuales resulta tan difícil hacer filosofía) a una conclusión que no se desprende de los principios que estamos examinando.

El programa de FpN apenas se está difundiendo en nuestro país, por lo que resulta prematuro hacer una evaluación de sus logros. Pero el Institute for the Advancement of Philosophy for Children ha venido trabajando durante más de treinta años en los Estados Unidos. Esto significa que muchos de los niños que se beneficiaron de este enfoque son hoy adultos críticos e independientes intelectualmente, que pueden aportar positivamente a las sociedades democráticas donde viven. Sería deseable, por lo tanto, conocer los resultados de las evaluaciones sistemáticas que se han hecho sobre los beneficios obtenidos mediante este programa en esos países. En particular, valdría la pena determinar qué tanto ha contribuido la $\mathrm{FpN}$ en la consecución de sus meta-objetivos a nivel político y ético, y qué proporción del mejoramiento de las habilidades de pensamiento superior, del establecimiento de una moralidad aceptable para la sociedad y del desarrollo adecuado en el dominio de lo afectivo y actitudinal, puede atribuirse a la influencia directa del programa sobre los alumnos y a su influencia indirecta a través del tratamiento filosófico de las demás asignaturas del currículo.

10 Ibíd., p. 6. 


\section{Los riesgos del programa de $\mathrm{FpN}$}

Propongo clasificar los riesgos a los que se enfrenta el programa de $\mathrm{FpN}$ en tres categorías. La primera, conformada por los riesgos provenientes de la formación filosófica y pedagógica de los encargados de orientar las asignaturas de la $\mathrm{FpN}$, al igual que los relacionados con la formación de quienes deben evaluar su trabajo y avalar los resultados del programa. La segunda, en la que debemos incluir los riesgos a que exponemos a los niños a quienes formamos de acuerdo con los lineamientos de esta propuesta, y de paso a quienes no formamos según tales lineamientos bajo el supuesto de que, en efecto, la FpN realmente consigue sus objetivos y ofrece mejores resultados que cualquier otro enfoque o método pedagógico disponible. La tercera, que se deriva de los riesgos que genera el cultivo de la mayor parte de las asignaturas del área de las humanidades. Explicaré brevemente cada una de estas categorías.

Es evidente que profesores de filosofía (e incluso de otras áreas) mal preparados o mediocres podrían sentirse inclinados a abrazar la $\mathrm{FpN}$ pues les proporciona un medio relativamente seguro para esconder sus limitaciones. De igual modo, si los evaluadores del programa o de sus resultados tienen falencias académicas parecidas, la situación puede tornarse en dramática ${ }^{11}$. En tanto la $\mathrm{FpN}$ se cultiva en círculos muy reducidos y donde hay poca crítica externa, parece ser un área particularmente vulnerable a la mediocridad, la falta de rigor y la perpetuación del error. Puede alegarse que, ya que la $\mathrm{FpN}$ no tiene como objetivo la enseñanza de la filosofía sino el desarrollo de habilidades superiores de pensamiento, este riesgo no existe y que, por tanto, la posibilidad de que se perpetúen ciertos errores doctrinales es muy baja. Tal vez esto sea cierto, pero siempre es posible que junto a los

11 Diego Antonio Pineda anticipa una objeción parecida. Ver su ponencia: "Algunas observaciones sobre el desarrollo de 'Filosofía para niños' en Colombia”. En: Memorias III Congreso Latinoamericano de Filosofia para niños, 2004, pp. 8-9. errores conceptuales se deslicen errores de lógica y razonamiento que los niños tenderían a aprender con entusiasmo, como el producto legítimo de las comunidades de indagación. Para resumir este punto, basta con recordar que el error nos acecha en cada esquina y que las precauciones para reconocerlo y evitarlo nunca serán excesivas.

Por lo que concierne a la segunda categoría, es posible echar a perder las mentes de los niños mediante deformaciones indeseables. No sostengo que los niños sean intrínsecamente incapaces de involucrarse en actividades racionales o de pensar correctamente, pero dudo que puedan razonar con los niveles de complejidad que requiere la filosofía, tras unos cuantos años en las "comunidades de indagación". Con todo, esta afirmación puede ser refutada por la experiencia y esperaré gustoso un contraejemplo $^{12}$. Lo que realmente me preocupa es que se fomente una idea falsa entre los niños, una idea que los persuada de que, en efecto, hacen filosofía cuando aprenden a usar fluidamente determinados vocablos de su lenguaje. También me perturba que el supuesto hecho de "hacer filosofía" sea motivo de alarde entre aquellos niños que participan del programa, como lo confiesan sus mismos creadores. Por otro lado, y teniendo en cuenta que, al menos en Colombia, el programa ha calado sobre todo en escuelas y colegios privados donde se educan los niños de las clases más altas de la sociedad, si la FpN realmente consigue sus objetivos, entonces podríamos promover inequidades intolerables al abstenernos de ponerlo en práctica con ciertos niños (por ejemplo, con los que asisten a las escuelas públicas).

La última categoría resulta todavía más preocupante. Si la FpN es una moda académica, entonces estaríamos promoviendo las veleidades de lo novedoso y en nombre de principios y fines educativos

\footnotetext{
12 Me refiero a un contraejemplo genuino y no a la interpretación piadosa y exageradamente favorable que hacen Lipman y sus amigos de las frases enigmáticas que suelen pronunciar los niños, participen o no de "comunidades de indagación".
} 
de reconocida importancia, despilfarrando tiempo y recursos. Pero incluso si el programa resulta no ser una moda, es preciso guardarnos de riesgos como el adoctrinamiento, la incompetencia disfrazada de profundidad, el verbalismo, la hipocresía académica, la pedantería y la ingenuidad. Como lo he repetido tantas veces, la fascinación que ejerce sobre casi todos nosotros la palabra, y la rapidez con que ciertas personas consiguen un dominio notable sobre los recursos del lenguaje, pueden fácilmente degenerar en discursos llenos de trivialidades, despropósitos y falsedades, que son saludados con entusiasmo por los legos y celebrados con los merecimientos de las más sólidas piezas del pensamiento racional por variados auditorios. Las comunidades de indagación y la promesa de hacer filosofía con los niños resultan ser candidatos naturales para la proliferación de semejantes vicios.

\section{La responsabilidad de los departamentos de filosofía}

Quienes nos dedicamos al cultivo de la filosofía tenemos la responsabilidad de examinar críticamente no sólo los planteamientos de índole estrictamente filosófico, sino también aquellas propuestas relacionadas con la enseñanza de la filosofía y con el rol que se le atribuya o reconozca a esta disciplina en el campo de la educación. Dado que la FpN tiene implicaciones en todos estos frentes, resulta necesario emprender cuanto antes un estudio juicioso de la naturaleza de su programa, de sus alcances y de sus implicaciones; de sus estrategias y de sus recursos didácticos. En particular, tenemos que estudiar no sólo los fundamentos y los objetivos del programa, sino las evaluaciones sistemáticas que se han hecho de sus resultados, y las experiencias mediante las que se ha pretendido impulsar este programa en nuestro país. En el nivel teórico, por ejemplo, considero que la motivación del programa es bastante endeble. En efecto, Lipman y sus asociados identifican dos argumentos a favor de la FpN, uno de carácter amplio y otro más reducido. Según el primero, la $\mathrm{FpN}$ “representa paradigmáticamente la educación del futuro como una forma de vida que aún no ha sido puesta en práctica y como una clase de praxis"; de acuerdo con el segundo, la FpN realiza "una contribución al currículo actual y al aula". Pero como se puede ver fácilmente, el segundo argumento es precisamente un supuesto que hay que probar y el primero se parece notablemente a un deseo o cuando mucho a un argumento de concebilidad. Que la educación del futuro incorpore masiva y activamente a la filosofía es algo que desearíamos todos por igual, pero por ahora sólo constituye una de las promesas más esperanzadoras que nos hacen los partidarios de la FpN.

Este breve ejemplo debe servir para ilustrar la necesidad de que profesores y estudiantes universitarios de filosofía dediquen más atención al tema de la FpN. Por encima del escepticismo que muchos de nosotros tenemos al respecto, debe estar la responsabilidad social de examinar una idea que puede convertirse súbitamente en práctica generalizada en un país como el nuestro que, frente al fracaso escolar y la mediocridad del sistema educativo, tiende a regirse por las modas pedagógicas y está siempre ávido de ensayar soluciones llamativas, sin detenerse a examinar seriamente si son plausibles o no.

\section{Conclusiones}

Los defensores del programa de FpN no han logrado estructurar un caso contundente a favor de su propuesta. Lipman y sus discípulos parten del supuesto de que el modelo escolar tradicional ha fracasado ${ }^{13}$ (en sentido estricto este fracaso no puede ser total pues, a lo menos, ellos mismos consiguieron advertir el problema y desarrollar las habilidades

\footnotetext{
13 Conviene señalar que el modelo de educación "tribal" (caracterizado por ser autoritario y obtener pobres resultados en el desarrollo de las habilidades de pensamiento superior, entre otros objetivos) contra el que reaccionan Lipman y sus seguidores no es otra cosa que un estereotipo superado hace ya mucho tiempo.
} 
superiores de pensamiento hasta los estándares adecuados para diseñar estrategias de solución) e identifican una serie de aspectos particulares en los que vale la pena buscar niveles crecientes de calidad. En términos generales, todos deseamos una educación encaminada a formar individuos analíticos, críticos, curiosos, creativos, dispuestos a usar sistemas adecuados de reglas morales, propensos a convertirse en buenos ciudadanos, tolerantes y de mente abierta, etc. Pero, ¿por qué llamar "filosofía" al conjunto de estrategias y prácticas educativas con las que se asegura el cumplimiento de dichos objetivos? Es cierto que hay muchos elementos filosóficos en el conjunto de postulados y estrategias que el programa de FpN recomienda, pero de ahí a considerarlos como un equivalente de la filosofía hay una distancia apreciable que tiene que ser llenada con algo más que el deseo y la ingenuidad de profesores y administradores escolares.

Reconozco que los profesores y estudiantes universitarios necesitamos estudiar más y mejor los pormenores del programa de $\mathrm{FpN}$, y examinar cuidadosamente las evaluaciones y los reportes periódicos de progreso. También acepto que no estamos investidos de ninguna autoridad especial (que pudiera ser necesaria para avalar el programa), y que muchos desarrollos filosóficos y educativos pueden ocurrir en el mundo mientras estamos distraídos en discusiones cerradas que interesan sólo a los especialistas. Como contraprestación espero que los partidarios de la FpN acepten, a su vez, que sus propuestas son susceptibles de crítica y examen racional y que, por lo menos lo concerniente a los aspectos puramente filosóficos del programa (y lo relativo a los contenidos), tiene que ser discutido con los filósofos profesionales. Desde este punto de vista, sería interesante que los departamentos de filosofía acompañaran el proceso de redacción y prueba de los materiales filosóficos no convencionales con que se adelanta el programa. Sería conveniente, también, evaluar las novelas filosóficas que se emplean en FpN y, tal vez, determinar qué tan eficaces resultan a la hora de enseñar contenidos filosóficos que consideramos importantes. También creo indispensable explorar la posibilidad de redactar novelas filosóficas propias, fieles a nuestras realidades, pero respetuosas de los contenidos filosóficos primordiales, en lugar de limitarnos a traducir lo que se ha escrito para los niños estadounidenses.

El programa de FpN propone un currículo que se puede desarrollar desde los primeros años de la escuela elemental y que, naturalmente, comienza con ejercicios de consideración de roles o situaciones que requieren algún tipo de debate y selección de alternativas. Encuentro curioso que a pesar de todos los argumentos para rechazar tesis como las de la psicología evolutiva, y de paso desacreditar las consecuencias de dichas tesis para la organización y presentación didáctica de material filosófico, los partidarios de la FpN terminan difiriendo la consideración de temas como la epistemología o la ontología a los niveles más avanzados de la escuela (tal y como se ha venido haciendo hasta ahora), e introducen los temas y problemas filosóficos de su programa de una manera secuencial, notablemente reminiscente de las recomendaciones que se han formulado desde hace mucho tiempo desde la pedagogía y la teoría instruccional ${ }^{14}$.

Para finalizar, permítanme reiterar la importancia de involucrarnos activamente en la discusión sistemática de los problemas que origina el pro-

\footnotetext{
${ }^{14}$ El currículo está organizado por etapas con objetivos claramente identificados y materiales (novelas filosóficas) para acompañar el trabajo de maestros y estudiantes. De preescolar hasta los 7 años se trabaja en la adquisición del lenguaje, y al reconocimiento de formas de razonamiento que están implícitas en la conversación diaria de los niños; en esta etapa se emplea la novela Elfie, y el manual Relacionando nuestros pensamientos; de 8 a 9 años, se prepara a los niños para que en la etapa siguiente comiencen a explorar el razonamiento formal, se emplea la novela Kio y Guss, y el manual Asombrándose ante el mundo, y tal vez también se acuda a la novela Pixie y al manual En busca del sentido. De 10 a 11 años se hace énfasis en la adquisición de la lógica formal e informal, mediante la novela El descubrimiento de Harry Stottlemeier, y el manual Investigación filosófica. Con los niños de 11 años se discuten los conceptos básicos de la ciencia, como objetividad, predicción, verificación, medida y causalidad, entre otros, con ayuda de la novela Tony y el manual de Investigación cientifica. De 12 a 14 años se tratan temas como la investigación ética, el lenguaje y los estudios sociales. El material lo constituye una novela titulada
} 
grama de FpN. Y para hacer justicia al título de mi ensayo, permítanme aclarar que, en mi opinión, este proyecto no representa de ningún modo una amenaza para los estudiantes y los profesores universitarios de filosofía. No creo que los miembros de los departamentos universitarios de filosofía debamos albergar ningún temor hacia la FpN. Si el proyecto resulta exitoso, en menos de lo que tardará el relevo generacional tendremos una nueva clase de estudiantes excepcionalmente dotados para hacer filosofía y favorablemente inclinados a extender los métodos de análisis y reflexión crítica de la filosofía a todos los campos del saber, con lo que no sólo conseguiremos elevar notablemente

\section{Bibliografía}

BLANCO, M. (2004). "Filosofía con niños". En: Filosofía, pedagogía y enseñanza de la filosofía. Universidad Pedagógica Nacional.

LIPMAN, M. (1969), (2000). El descubrimiento de Harry. Novela de lógica formal. Traducción y adaptación cultural para Colombia de Diego Pineda, Bogotá.

, SHARP A. M. y OSCANYAN, F. S. (1992). La Filosofía en el aula, Madrid, Ediciones de la Torre. los estándares de la discusión filosófica en nuestro país, sino también influir positivamente en todo el proceso educativo. Si el proyecto no funciona, a lo menos podremos advertir oportunamente a la comunidad educativa sobre sus riesgos y diseñar estrategias para aminorar sus efectos indeseables. Por contraste, se me antoja pensar que los partidarios de la FpN y quienes se encuentran involucrados activamente en su fortalecimiento en nuestro país, sí tienen motivos para temer. Deben temer a todos los riesgos que entraña la aplicación indiscriminada, incompetente y superficial de sus principios al currículo mediante el que educamos nuestros niños.

PINEDA, D. A. (2004). "Algunas observaciones sobre el desarrollo de Filosofía para niños en Colombia". En: Memorias III Congreso Latinoamericano de Filosofía para niños, pp. 8-9.

(2003). "Filosofía para niños: un Proyecto de Educación Filosófica”. En: Cultura. Separata No. 3, pp. 1-20. 